\title{
Fundamental Properties of Magnetization
}

\section{OVERVIEW}

In UNIT B1.1 and UNIT B1.2, we discussed the spin properties of the proton by considering one spin with a magnetic moment $\vec{\mu}$. We also introduced the relation between the magnetic moment and the angular momentum. In this unit, the concept of bulk magnetization and quantized spin angular momentum are introduced. The dependence of the magnetization on spin density, temperature and magnetic field is presented.

Historically, it was Stern and Gerlach in the early 1920s who discovered that angular momentum is quantized. They shot a beam of neutral silver atoms through a vertical magnetic field (see Fig. B1.3.1). Surprisingly, the silver beam was not simply deflected by the magnetic field. Instead, the beam split into two beams corresponding to two different values of the spin angular momentum of the electron. Further experiments showed that the proton also has spin one-half and the same splitting effect. This effect is due to the discretized energy levels of the spins and it is an example of the general Zeeman splitting effect (see Fig. B1.3.2).

The magnetic moment vector for a typical proton is prevented from relaxing fully to an alignment along the external magnetic field because of the thermal energy associated with the absolute temperature $T$. From the technical discussion below, we can compare the magnetic field interaction with the average thermal energy $k T$, where $k$ is the Boltzmann's constant. At human body temperatures, the thermal energy is millions of times larger than the quantum energy difference for parallel alignment (lower energy) versus antiparallel alignment (higher energy) of the spin. For a proton with only two quantum spin states, these are the only two possible alignments. The energy difference between these two states is $\Delta E=\hbar \omega$ where $h$ is Planck's constant and $\hbar \equiv h /(2 \pi)$. Significantly, the frequency in this energy difference between these two states, $\omega_{0}$, is nothing other than the Larmor precession frequency.

The extreme smallness of the quantum spin energy compared with the thermal energy means that the fraction $\hbar \omega_{0} /(k T)<<1$. In that case, the Boltzmann probability (see Technical Discussion) demonstrates why the number of spins parallel to the magnetic field exceeding the number anti-parallel to that field, the "spin excess," is also very small. Specifically, the spin excess is limited by a factor involving that fraction:

$$
\text { spin excess } \simeq N \frac{\hbar \omega_{0}}{2 k T}
$$

where $N$ is the total number of spins present in the sample. It is found that the spin excess is only one in a million spins even for a magnetic field strength as large as $0.3 \mathrm{~T}$.

Since the spin excess is millions of times smaller than the total number of proton spins, it might be guessed that no significant signal would be detected at room temperature. However, there are Avogadro numbers of protons in a few grams of tissue. Consider the average of the volume density of the magnetic moment, or "longitudinal equilibrium magnetization" $M_{0}$ for the component of the magnetic moment vector along the external field direction. For a sample with $\rho_{\text {den }}$ defined as the number of protons per unit volume (or the "spin density"), the longitudinal equilibrium magnetization is given by the proton magnetic moment component $\gamma \hbar / 2$ multiplied by the relative spin excess in Equation B1.3.1 times the spin density. Using the Larmor equation, the available bulk magnetization is given by:

Contributed by Yu-Chung Norman Cheng and E. Mark Haacke

Spin Behavior

Current Protocols in Magnetic Resonance Imaging (2001) B1.3.1-B1.3.9

B1.3.1

Copyright $@ 2001$ by John Wiley \& Sons, Inc.

Supplement 1 


$$
M_{0}=\frac{\rho_{d e n} \gamma^{2} \hbar^{2}}{4 k T} B_{0}
$$

This equilibrium value, while limited by the spin excess, leads to measurable NMR effects. Of particular note is the linear dependence of $M_{0}$ with $B_{0}$ and the quadratic dependence of $M_{0}$ with the gyromagnetic ratio $\gamma$. Fortuitously, the high value of $\gamma$ for protons and its high spin density make it the most attractive nucleus to image.

Practically, the detected signal is proportional to the magnetization of an ensemble of spins (a spin isochromat). Thus, we can simply replace the magnetic moment with the magnetization in many equations such as the Bloch equations. The beauty is that the physics concepts behind our early discussions of energy, forces, and the equations of motion do not change, whether we consider a single spin or a set of spins. The reader will see this in the following technical section. The full signal dependence on $B_{0}$ and $\gamma$ will only become apparent in Chapter B2.

\section{TECHNICAL DISCUSSION}

\section{Magnetization Vector}

For images of a macroscopic body, we focus on protons, introducing their local magnetic moment per unit volume, or magnetization, as $\vec{M}(\vec{r}, t)$. Consider a volume element ("voxel") with volume $V$ small enough that external fields are, to a good approximation, constant over $V$, but big enough to contain a large number of protons. The external fields are assumed to vary spatially only over scales much larger than $V^{1 / 3}$. The bulk magnetization is then defined through the expression:

$$
\vec{M}=\frac{1}{V} \sum_{\substack{\text { protons } \\ \text { in V }}} \vec{\mu}_{i}
$$

The set of spins in $V$ is called a spin "isochromat," which can be defined as an ensemble or domain of spins with the same phase. Disregarding the interactions of the protons their environment, a sum over the (Equation B1.1.20) yields:

$$
\frac{1}{V} \sum_{i} \frac{d \vec{\mu}_{i}}{d t}=\frac{\gamma}{V} \sum_{i} \vec{\mu}_{i} \times \vec{B}_{e x t}
$$

or

$$
\frac{d \vec{M}}{d t}=\gamma \vec{M} \times \vec{B}_{e x t} \quad \text { (non-interacting protons) }
$$

It is most advantageous to analyze the magnetization, and its differential equation, in terms of parallel and perpendicular components defined relative to the static main magnet field, $\vec{B}_{\text {ext }}=B_{0} \hat{z}$. The parallel, or "longitudinal" component of the magnetization is:

$$
M_{\|}=M_{z}
$$

The transverse components are:

$$
\vec{M}_{\perp}=M_{x} \hat{x}+M_{y} \hat{y}
$$


The corresponding components of the cross-product in Equation B1.3.5 lead to the decoupled equations:

$$
\frac{d M_{z}}{d t}=0 \quad \text { (noninteracting protons) }
$$

and

$$
\frac{d \vec{M}_{\perp}}{d t}=\gamma \vec{M}_{\perp} \times \vec{B}_{\text {ext }} \quad \text { (noninteracting protons) }
$$

The modeling of the proton interactions with their neighborhood leads to additional terms in Equation B1.3.8 and Equation B1.3.9 which depend on decay parameters, and these parameters are different in the two equations. This difference is related to the fact that, in contrast to a given magnetic moment, the magnitude of the macroscopic magnetization is not fixed, since it is the vector sum of (many) proton spins. The components of $\vec{M}$ parallel and perpendicular to the external field "relax" differently in their approach to equilibrium.

Equation B1.3.8 is certainly wrong for interacting protons, insomuch as their moments try to align with the external field through the exchange of energy with the surroundings. To understand the origin of the missing term, an energy argument is helpful.

\section{Potential Energy}

The classical formula for the potential energy associated with a magnetic moment immersed in a magnetic field is:

$$
U=-\vec{\mu} \cdot \vec{B}
$$

This implies that the moment will tend to line up parallel to the field in order to reach its minimum energy state, if energy can be transferred away. Since the protons are considered to be in thermal contact with the lattice of nearby atoms, the thermal motion present in the lattice can account for any change in a given proton spin energy (Equation B1.3.10). In the quantum language, a spin can exchange a quantum of energy with the lattice.

The magnetization version of Equation B1.3.10 is the potential energy density:

$$
U_{M}=-\vec{M} \cdot \vec{B}=-M_{\|} B_{0}
$$

involving only the longitudinal component of the magnetization. Although the transverse components can be ignored in discussing the energy, it follows that, as the longitudinal magnetization returns to its equilibrium value $M_{\mathrm{o}}$, the transverse magnetization must vanish. In fact, the transverse magnetization can vanish more quickly due to "dephasing" (see Chapter B3). 


\section{Discrete Angular Momentum and Energy}

Discreteness of the energy levels of the proton's magnetic moment interaction with a magnetic field is related to the discreteness of the proton's intrinsic angular momentum, or spin. In fact, this is the historical path along which scientists came to the conclusion that spin was quantized. In the early 1920s, Stern and Gerlach experimented with a horizontal beam of neutral silver atoms passing through a perpendicular (vertical) magnetic field gradient (see Fig. B1.3.1). Even with zero electric charge for the atom, a magnetic force is exerted on any atom that possesses a nonzero magnetic moment, as is the case for a silver atom, in a spatially varying magnetic field.

To see this, an expression for the force can be found from the gradient of the magnetic potential energy $U$ in Equation B1.3.10. For a field $\vec{B}$,

$$
\vec{F}=-\vec{\nabla} U=\vec{\nabla}(\vec{\mu} \cdot \vec{B})
$$

The field produced by the magnet in the figure has $y$-and $z$-components, and both are spatially varying. But, averaged over time, the $y$-component of the magnetic moment (and its spatial gradient) in the central region is zero. This follows from the expected classical precession about the $z$-axis. Therefore, $\vec{\mu} \cdot \vec{B}$ may be replaced by $\mu_{z} B_{z}$ in Equation B1.3.12, and, since $\vec{\mu}$ is independent of position, the $z$-component of Equation B1.3.12 becomes:

$$
F_{z}=\mu_{z} \frac{\partial B_{z}}{\partial z}=\mu_{z} G_{z}
$$

The notation $G_{z}$ for the $z$-derivative of the vertical component of the field gradient will be useful in imaging discussions about "field gradients."

The experiment of Stern and Gerlach had the two necessary ingredients entering into Equation B1.3.13, gradients and moments. They used electromagnets with vertical pole faces where the $z$-component of the field increases as the upper pole is approached (Fig. B1.3.1A). The angular momentum of an unpaired electron in a silver atom gives rise to an atomic magnetic moment whose component parallel to the field gradient would lead to a deflection according to Equation B1.3.13. (In this case, the nuclear magnetic moment is negligible; the reader is referred to the discussion in UNIT B1.1.) The relation analogous to Equation B1.1.17 is:

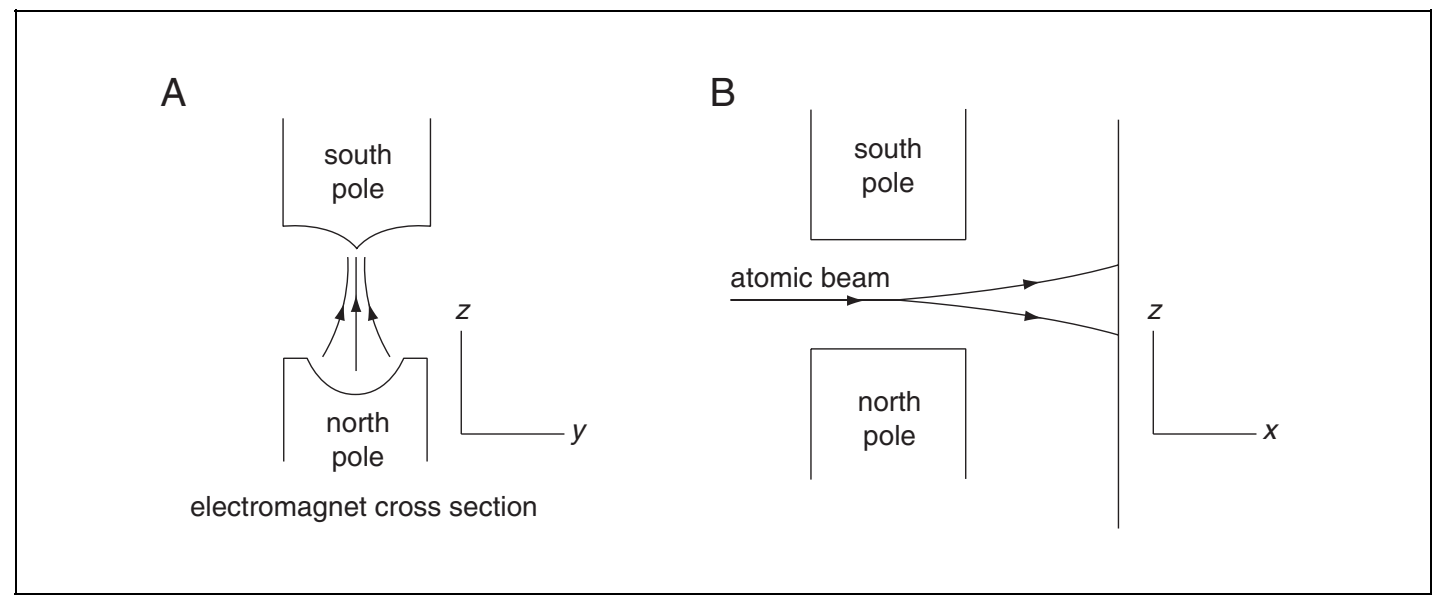

Figure B1.3.1 The historical Stern-Gerlach experiment: Using a vertical ( $z$-axis) magnetic field gradient from an electromagnet to split a beam of silver atoms in the direction of the gradient. Two views of the experiment are shown. In (A), the direction of the beam is out of the page. In $(\mathbf{B})$, a side view of the beam is shown. 


$$
\vec{\mu}_{e}=\gamma_{e} \vec{J}_{e}
$$

in terms of the spin angular momentum $\vec{J}_{e}$ associated with the electron.

The classical conclusion from Equation B1.3.13 and Equation B1.3.14 is that the beam should be deflected through a spread of angles, due to a continuous spread of magnetic moment values, or, more fundamentally, a continuous spread of angular momentum values. Working at the time when many profound quantum discoveries were being made, Stern and Gerlach had the specific goal of searching for quantization effects, and they found them. Instead of a smear of deflection angles, their measurements showed that the beam split vertically into two beams, corresponding to two discrete values for the $z$-component of the angular momentum of the electron. Although it was not known at that time, we now understand the angular momentum of the unpaired silver electron to be entirely due to its spin; there is no orbital angular momentum for this electron state. The spin component in Equation B1.3.13 must be quantized.

With the motivation given above, let us describe, as an experimental fact, the quantization of angular momentum for the spin vector $\vec{S}$. The magnitude of $\vec{S}$ satisfies:

$$
S^{2}=s(s+1) \hbar^{2} \quad \text { where } s=0, \frac{1}{2}, 1, \frac{3}{2}, \ldots
$$

with $2 s+1$ values of $m_{s}$ (i.e., $-s,-s+1, \ldots, s-1, s$ ) pertaining to the $z$-component $S_{z}=m_{s} \hbar$ of the spin vector and where $\hbar$ is Planck's constant $\left(h=6.626 \times 10^{-34} \mathrm{~J}\right.$-sec $)$ divided by $2 \pi$. The two deflection angles observed in the Stern-Gerlach experiment determine the spin quantum number $s$ for the electron. For the unpaired electron in a silver atom in the Stern-Gerlach experiment, it must be that $s=1 / 2$ and $m_{s}= \pm 1 / 2$. The electron has "spin one-half." At the time of the experiment, by the way, it was not known whether an electron had any spin at all. We see that the existence of spin itself is inferred from such measurements.

Experiments in the years following the work of Stern and Gerlach showed that the proton must have spin one-half as well. Thus the magnetic moment (Equation B1.1.17) is discretized and Equation B1.3.10 leads to discrete energy values:

$$
E=-\vec{\mu} \cdot \vec{B}=-\mu_{z} B_{z}=-\gamma m_{s} \hbar B_{z}
$$

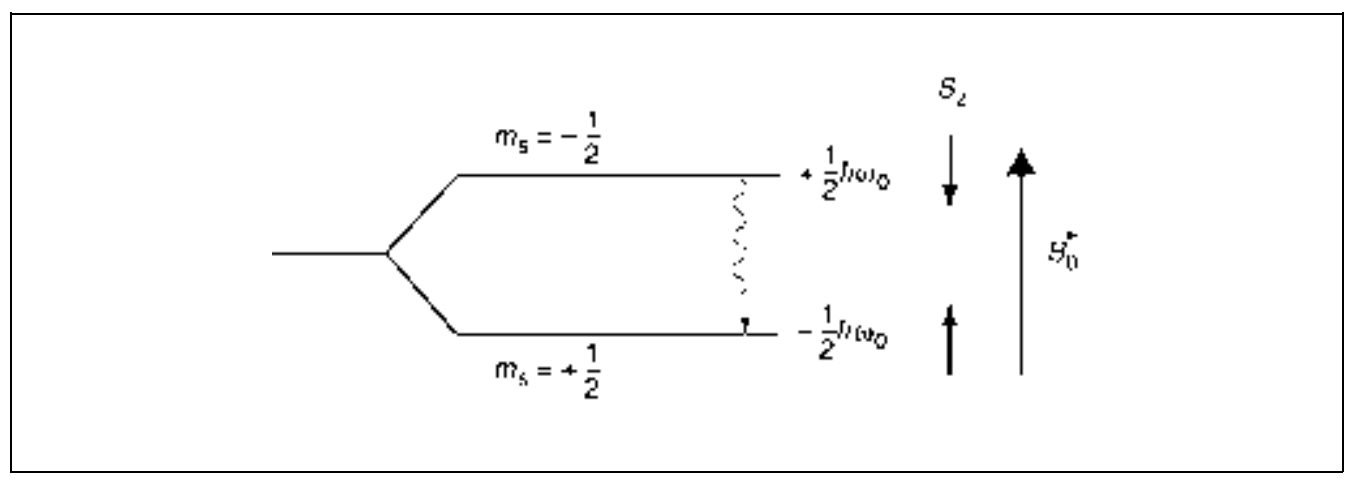

Figure B1.3.2 The Zeeman energy levels for a spin one-half system and a positive gyromagnetic ratio. The spin is parallel to the external field $B_{0} \hat{z}$ in the lower energy state. The wavy vertical line represents a transition from the higher to the lower state by photon emission.

Spin Behavior

\section{B1.3.5}

Supplement 1 
with

$$
m_{s}= \pm \frac{1}{2} \Rightarrow \begin{gathered}
\text { spin parallel to field } \\
\text { spin anti-parallel to field }
\end{gathered}
$$

The two $m_{s}$ values are also referred to as "spin-up" and "spin-down," respectively, stemming from a reference to the $z$ axis. The two proton energy levels predicted by Equation B1.3.16 are exhibited in Fig. B1.3.2. This is an example of the general Zeeman effect where atomic or nuclear magnetic moments in the presence of an external magnetic field lead to splittings in the atomic or nuclear energy levels.

\section{Boltzmann Equilibrium Values}

The equilibrium value $M_{0}$ was required in the solution of the Bloch equation for the longitudinal magnetization, $M_{z}(t)$. This value, arising in the relaxation limit $M_{z}(\infty)=M_{0}$, represents the trade-off between the tendency of a spin system to align itself with the external field (the lowest energy state), and its ability to gain energy from thermal contact.

If a particle was in thermal contact with other material, and at an absolute temperature of $T$, it would retain kinetic energy on the order of $k T$, where $k$ is Boltzmann's constant. The exchange interaction will leave this system (as well as a system of spins) in equilibrium somewhere above the (ground) state of lowest energy, depending on how large $k T$ is. The probability of finding a system with energy $\varepsilon$, while in contact with a much larger system (the "reservoir" depicted in Fig. B1.3.3) at a temperature $T$, is given by the normalized Boltzmann probability factor:

$$
P(\varepsilon)=\frac{e^{-\varepsilon / k T}}{Z}
$$

(See any introductory thermal physics text for a discussion of the Boltzmann factor.) The normalization divisor is the partition function, $Z$, the sum over all weighting factors:

$$
Z=\sum_{\varepsilon} e^{-\varepsilon / k T}
$$

A system of interest is a spin in thermal contact with the rest of a set of $N$ spins and with the background lattice all at a temperature $T$. The number $N$ is taken to be very large, along with the size of the lattice. To find the thermal equilibrium value of $M_{z}$, consider the calculation of the $z$-component of the average total magnetic moment for $N$ spins distributed over all possible magnetic spin states, neglecting translational motion. This brings together the thermal interactions and the quantum basis of the magnetization. The quantization axis is chosen along the external field direction (the $z$-axis, as usual) and the

Fundamental Properties of Magnetization

B1.3.6

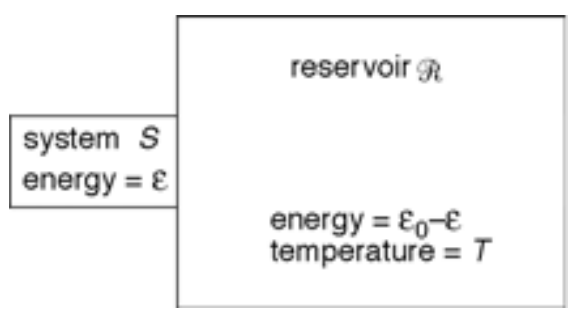

Figure B1.3.3 A small system in thermal equilibrium with a reservoir at temperature $T$. The total energy $\varepsilon_{0}$ is conserved between the two systems. 
case of a general spin $s$, with magnetic number $m_{\mathrm{s}} \equiv m$, is analyzed. The thermal average of the magnetization is

$$
M_{0}=\rho_{\operatorname{den}} \sum_{m=-s}^{s} P(\varepsilon(m)) \mu_{z}(m)
$$

where $\rho_{\text {den }}=N / V$ is the density of spins per unit volume in the homogeneous isochromat of volume $V$. From Equation B1.3.16 and $\omega_{0}=\gamma B_{0}$, where $B_{0}$ is the magnitude of the applied (external) magnetic field,

$$
\begin{gathered}
\varepsilon=-m \hbar \omega_{0} \\
\mu_{z}=m \gamma \hbar
\end{gathered}
$$

The explicit expression for the equilibrium magnetization is

$$
M_{0}=\left(\frac{N \gamma \hbar}{V}\right) \frac{\sum_{m=-s}^{s} m e^{m u}}{\sum_{m=-s}^{s} e^{m u}}=\left(\frac{N \gamma \hbar}{V}\right) \frac{s\left(1-e^{-(2 s+1) u}\right)-(s+1) e^{-u}\left(1-e^{-2 s u}\right)}{\left(1-e^{-u}\right)\left(1-e^{-(2 s+1) u}\right)}
$$

with

$$
u=\frac{\hbar \omega_{0}}{k T}
$$

Equation B1.3.23 can be simplified in MR, because the nuclear magnetic energies are so much smaller than room-temperature thermal energies. For human body temperature (310 $\mathrm{K}$ ), and protons, the basic exponent unit in Equation B1.3.23 and Equation B1.3.24 has the numerical value $u \cong 6.6 \times 10^{-6} B_{0}$ for $B_{0}$ in Tesla. Hence, the basic exponential is unity to within 10 parts per million for field strengths in that range, and we can make the following approximation:

$$
\begin{aligned}
\exp \left(\frac{\hbar \omega_{0}}{k T}\right) & =1+\frac{\hbar \omega_{0}}{k T}+\mathcal{O}\left(\left(\frac{\hbar \omega_{0}}{k T}\right)^{2}\right)+\ldots \\
& \simeq 1+6.6 \times 10^{-6} B_{0}
\end{aligned}
$$

For the finite $m$ values of interest, $e^{m u}$ is also very close to unity, leading to a simplified limit for Equation B1.3.23. For arbitrary spins, considering the expansion of small $x$ in $e^{-x}$ to the third order, Equation B1.3.23 yields:

$$
M_{0} \simeq \rho_{\mathrm{den}} \frac{s(s+1) \gamma^{2} \hbar^{2}}{3 k T} B_{0} \quad\left(\hbar \omega_{0} \ll k T\right)
$$


This form of $M_{0}$ will be used in UNIT B2.1 for defining the relative signal strengths of different elements in an MR experiment.

Equation B1.3.26 constitutes a quantum derivation of the experimental Curie's law for magnetization, which states that the magnetization should be proportional to $1 / T$, but with the bonus of determining the coefficient. For a spin one-half particle, such as proton $(s=$ 1/2), Equation B1.3.26 becomes:

$$
\left.M_{0} \simeq \frac{1}{4} \rho_{\text {den }} \frac{\gamma^{2} \hbar^{2}}{k T} B_{0} \quad \text { (spin one-half particle, } \hbar \omega_{0} \ll k T\right)
$$

Finally, let us use these results in terms of the proton spin excess, defined by the difference between the number of spins parallel $(N(\uparrow))$ and antiparallel $(N(\downarrow))$ to the external field:

$$
\begin{aligned}
\Delta N & \equiv N(\uparrow)-N(\downarrow) \\
& =N\left(P_{+}-P_{-}\right) \\
& \simeq \frac{N u}{2}
\end{aligned}
$$

where $N$ is the total number of spins in the system and the Boltzmann probability (Equation B1.3.18) for the two spin- $1 / 2$ states $\left(m= \pm \frac{1}{2}\right.$ or $\left.\varepsilon=\mp 1 / 2 \hbar \omega_{0}\right)$ is:

$$
P_{ \pm}=\frac{e^{ \pm u / 2}}{e^{u / 2}+e^{-u / 2}}
$$

In the applications to MRI, Equation B1.3.27 is very small compared to the maximum possible magnetization (which would be the product of the spin density times the individual spin magnetic moment). This is because the aforementioned parameter $u$ is very small. Since the proton spin energy, Equation B1.3.10, is tiny compared with the thermal energy scale $k T$ ( $k$ is the Boltzmann's constant and $T$ is in Kelvin) at room temperature, there is only a minuscule energy advantage for a spin moment to be aligned with the magnetic field. In consequence, only a very small fraction (about five in one million for a field strength of $1.5 \mathrm{~T}$ ) of parallel spins exceed anti-parallel spins for field strengths of interest. Fortunately, Avogadro's number is so large, that, for example, on the order of $10^{18}$ excess proton spins are aligned along a $0.5 \mathrm{~T}$ field in one mole of water. Hence, the magnetization $M_{0}$ is still big enough to be measured.

\section{KEY REFERENCES}

Abragam, A. 1961. The Principles of Nuclear Magnetism. Clarendon Press, New York.

Excellent reviews of the basic quantum aspects required to understand the NMR signal appear in this text.

Bloembergen, N., Purcell, E.M., and Pound, R.V. 1948. Relaxation effects in nuclear magnetic resonance experiments. Phys. Rev. 73:679.

The fundamental concepts of the quantum processes are given in this article.

Haacke, E.M., Brown, R.W., Thompson, M.R., and Venkatesan, R. 1999. Magnetic Resonance Imaging: Physical Principles and Sequence Design. John Wiley and Sons, New York.

This text covers the technical aspects presented here, but in more detail, and also discusses more advanced materials.

Purcell, E.M., Torrey, H.C., and Pound, R.V. 1946. Resonance absorption by nuclear magnetic moments in a solid. Phys. Rev. 69:37.

The fundamental concepts of the quantum processes are given in this article.

B1.3.8 
Rabi, I.I., Ramsey, N.F., and Schwinger, J. 1954. Rotating coordinates in magnetic resonance problems. Rev. Modern Phys. 26:167.

This article presents an outstanding discussion of NMR in rotating reference frames.

Slichter, C.P. 1990. Principles of Magnetic Resonance, 3rd ed. Springer Verlag, New York.

This text reviews the relevant general concepts of rotation and precession in classical mechanics and NMR.

Contributed by Yu-Chung Norman Cheng and E. Mark Haacke

Case Western Reserve University

Cleveland, Ohio

and The MRI Institute for Biomedical Research

St. Louis, Missouri 